\title{
Atom Probe Tomography with the Easier to Operate EIKOS ${ }^{\mathrm{TM}}$
}

\author{
Katherine P. Rice ${ }^{1}$, Yimeng Chen ${ }^{1}$, Robert M. Ulfig ${ }^{1}$, Dan Lenz ${ }^{1}$, Joseph Bunton ${ }^{1}$, Mike van Dyke ${ }^{1}$,
} David J. Larson ${ }^{1}$

\section{${ }^{1 .}$ CAMECA Instruments, Inc., 5470 Nobel Drive, Madison, WI 53711 USA.}

Atom probe tomography (APT) has been used for decades to obtain nanoscale chemical information. Hardware developments in recent years have increased the fraction (field of view) and total number (depth) of ions detected, improved specimen yield, and allowed for a wider range of material systems to be investigated. CAMECA $^{\circledR}$ continues this pattern of innovation by introducing a simplified and lower cost of ownership atom probe. The new EIKOS ${ }^{\mathrm{TM}}$ makes it possible for a wider range of institutions to include atom probe as a routine part of their materials characterization process.

The EIKOS ${ }^{\mathrm{TM}}$ atom probe shown in Figure 1a provides atomic scale chemical information with a simplified design and a smaller footprint than the LEAP 5000 ${ }^{\mathrm{TM}}$ (Local Electrode Atom Probe) [1][2]. By focusing on traditional atom probe applications such as metallurgy, the EIKOS ${ }^{\mathrm{TM}}$ can accommodate many types of electropolished or FIB prepared samples in a wire-based geometry. The EIKOS ${ }^{\text {TM }}$ comes equipped with an optional laser, allowing a wider range of applications than voltage-only atom probes, as well as a reflectron flight path that allows for high mass resolving power and signal-to-noise ratio. Each specimen is pre-aligned outside of the vacuum system to an integrated local electrode (ILE) as shown in Figures 1b and 1c, which allows for longer lasting electrodes and lower cost overall. Additionally, the wire geometry used for specimens is ideal for correlative microscopy work such as TEM, transmission EBSD, or STEM, to provide a complete materials characterization story and enable faster development of new materials for industrial applications. Like all atom probe techniques, the EIKOS $^{\text {TM }}$ has an equal sensitivity to all isotopes and elements.

The EIKOS ${ }^{\text {TM }}$ uses a flat disk counter electrode with an aperture with a wire-geometry specimen (i.e. not a microtip carrier) aligned slightly protruding through the ILE plane (Figure 2a) [3]. Substantial field enhancement is possible, even while using relatively large apertures $(\sim 1 \mathrm{~mm})$, which minimizes the chance of damage to the electrode during specimen fracture events (Figure 2b). For a $\sim 1 \mathrm{~mm}$ aperture in a disc electrode, the field enhancement is $\sim 25 \%$ less than in a LEAP $^{\circledR}$ and is relatively insensitive to specimen penetration distance. Alignment to the electrode is completed outside the vacuum on a dedicated alignment station. Aligning a specimen within the counter electrode aperture takes approximately 2 minutes for an experienced user, and this configuration eliminates the need for a movable stage within the ultra-vacuum analysis chamber.

Applications for EIKOS ${ }^{\mathrm{TM}}$ encompass a wide range of materials. Grain boundary segregation, cluster finding, III-V materials, high entropy alloys, and multilayer magnetic thin films are all part of the applications space for EIKOS ${ }^{\text {TM }}$. Figure $3 \mathrm{a}$ and $3 \mathrm{~b}$ show ion maps of (a) a Ni-based superalloy showing carbon (dark red), titanium (dark green), and boron (blue) precipitates and (b) $\mathrm{Cr}$ rich $\gamma$ phase and clusters in a superalloy prepared by electropolishing. The base EIKOS ${ }^{\mathrm{TM}}$ model with voltage pulsing is appropriate for most metallurgical samples, while the optional laser enables applications such as oxides and semiconductor materials. 


\section{References:}

[1] S. S. Bajikar et al., Appl. Surf. Sci. 94/95 (1996) p. 464.

[2] D.J. Larson et al., "Local Electrode Atom Probe Tomography", (Springer, New York) (2013).

[3] R. Schlesiger, et al., Review of Scientific Instruments, 81 (2010), p. 043703.

$\mathbf{a}$

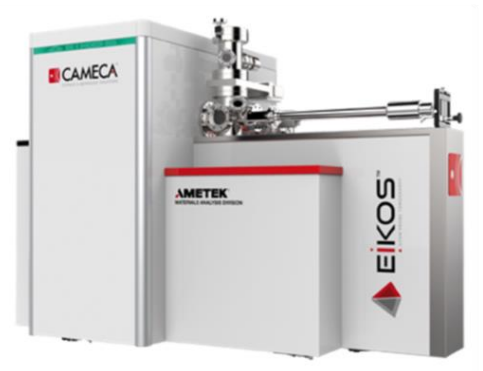

b

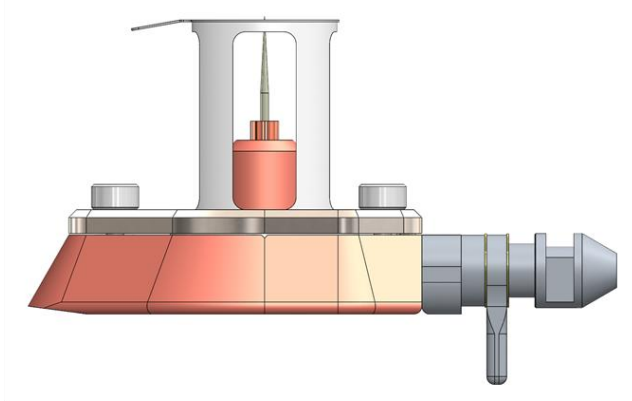

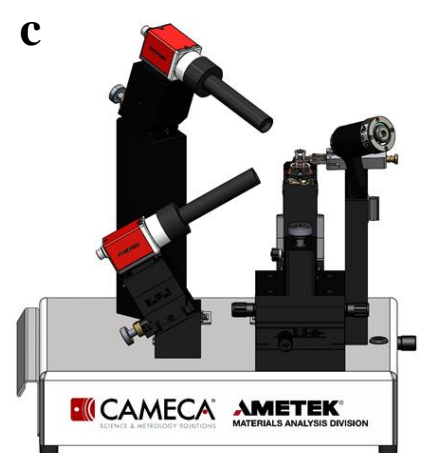

Figure 1. (a) EIKOS atom probe; (b) Integrated Local Electrode with wire specimen; (c) ex-situ alignment station schematic

$\mathbf{a}$

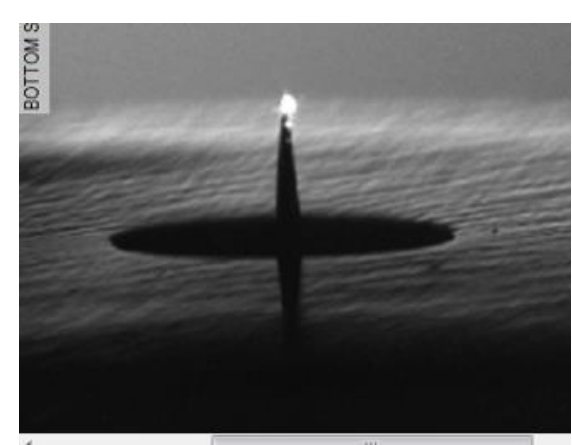

b

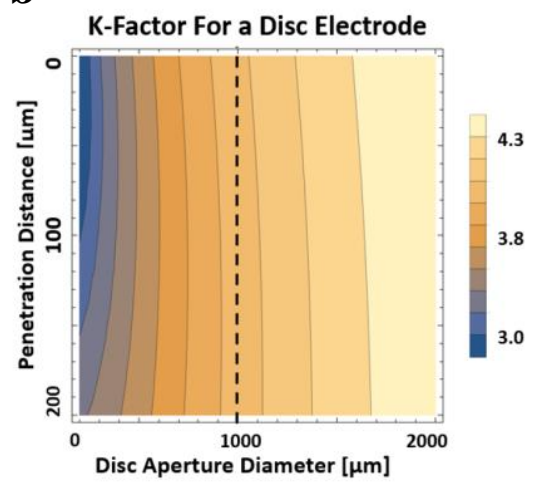

a

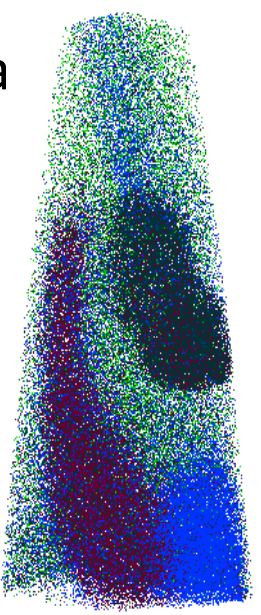

$\underline{20 \mathrm{~nm}}$

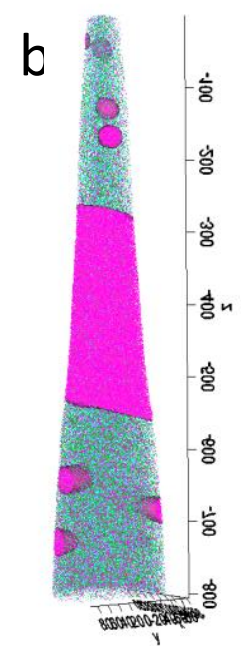

Figure 3. (a) ion map of Inconel 600 alloy with a grain boundary showing B segregation, and multiple precipitates; (b) Microstructure of $\mathrm{Ni}$ based superalloy 